\title{
Análise comparativa dos parâmetros de segmentação e regras de classificação das vias pavimentadas de Marília/SP
}

\section{Comparative analysis of segmentation parameters and classification rules for paved roads in Marília / SP}

\author{
Agnes Silva de Araujo \\ Mestre em Geografia, doutoranda em Geografia Física - FFLCH - USP, Brasil \\ agnes.araujo@usp.br \\ Isabela do Lago Silva \\ Graduanda em Geografia - FFLCH - USP, Brasil \\ lagoisa@usp.br \\ Beatriz Pereira \\ Graduanda em Geografia - FFLCH - USP, Brasil \\ beatriz2.pereira@usp.br
}

Alfredo Pereira de Queiroz Doutor em Engenharia, professor do Departamento de Geografia - FFLCH - USP, Brasil $\underline{\text { aqueiroz@usp.br }}$

\begin{abstract}
Resumo
Nos últimos anos, as técnicas de extração de informações das imagens de satélite evoluíram muito, mas o compartilhamento das suas regras de classificação ainda está muito aquém do seu potencial. Este artigo compara duas classificações de vias pavimentadas, desenvolvidas através do uso de técnicas de GEOBIA, por dois operadores independentes, de Marília-SP. A imagem do satélite WordView-II (RGB, NIR) de 2010 e o programa eCognition foram utilizados por ambos os intérpretes no processo. O coeficiente kappa, produzido a partir de 500 pontos aleatórios, e a análise visual foram utilizados para comparar as classificações. Os índices kappa de 0,68 e 0,72 mostraram considerável concordância entre os operadores. Ressaltaram também a importância dos critérios de segmentação e dos parâmetros de escala de análise, principais diferenças entre ambos, para a classificação dos objetos geográficos.
\end{abstract}

Palavras-chave: Análise de imagens baseadas em objetos geográficos (GEOBIA), vias pavimentadas, cobertura da terra, regras de classificação.

\begin{abstract}
In recent years, satellite imagery information extraction techniques have significantly evolved, but sharing their classification rules is still far below their potential. This paper compares two classifications of paved roads, developed through the use of GEOBIA techniques, by two independent operators, in Marília-SP. Both interpreters used the WordView-II satellite image (RGB, NIR) 2010 and the eCognition program in the process. The kappa coefficient, produced from 500 random points, and visual analysis were used to compare classifications. The kappa indexes of 0.68 and 0.72 showed considerable agreement among the operators. They also emphasised the importance of the segmentation criteria and the parameters of the scale of analysis, the main differences between the two, for the classification of geographic objects.
\end{abstract}

Keywords: Geographic object-based image analysis (GEOBIA), paved roads, land cover, classification rules. 


\section{INTRODUÇÃO}

Com o avanço das geotecnologias das décadas recentes, houve um significativo aprimoramento das aplicações de Sensoriamento Remoto. O uso dos mecanismos semiautomatizados para a extração de informações, por exemplo, contribuiu para aumentar a precisão e reduzir o tempo e os custos de manipulação dos dados (ALMEIDA, 2010).

A análise de imagens baseadas em objetos geográficos (Geographic Object-Based Image Analysis - GEOBIA) utiliza métodos semiautomatizados para classificar imagens do Sensoriamento Remoto (HAY; CASTILLA, 2008). O princípio da GEOBIA consiste em estabelecer um agrupamento de pixels e atribuir informações semânticas a esses grupos (BLASCHKE; HAY, 2001). A análise dos objetos geográficos de uma imagem pode então ser realizada de acordo com seus atributos geométricos, texturais e contextuais, além do comportamento espectral. Com a seleção dos parâmetros de escala, é possível avaliar a relação de vizinhança e homogeneidade entre pixels, e caracterizar objetos espacialmente contínuos (DESCLÉE et al,. 2006).

Inúmeras pesquisas demonstraram que as classificações por GEOBIA apresentam melhores resultados se comparadas às classificações pixel-a-pixel (MYINT et al., 2011, PLATT; RAPOZA, 2008, WHITESIDE; AHMAD, 2005, ORUC et al., 2004, CIVCO et al., 2002). Willhauck (2000), por exemplo, demonstrou a maior acurácia das classificações por GEOBIA $(94,7 \%)$ e redução das manchas descontínuas, em comparação com as classificações pixel-a-pixel, cujo resultado foi de $90,95 \%$. Outra vantagem importante é que os resultados da GEOBIA podem ser facilmente incorporados aos Sistemas de Informações Geográficas (SIG), ao contrário das classificações pixel-a-pixel, permitindo análises espaciais integradas (BLASCHKE; KUX, 2007).

Para Gardin et al. (2010), a classificação de objetos nas imagens de Sensoriamento Remoto está sujeita à subjetividade, razão pela qual distintos intérpretes geram classificações diferentes. Contudo, as regras de classificação por GEOBIA minimizam este aspecto. Elas podem ser compartilhadas, pois estão fundamentadas no conhecimento prévio do operador sobre as características geométricas, espectrais e texturais dos objetos geográficos (BELGIU et al., 2014). Conforme Mahdavi et al. (2018), é através do compartilhamento das regras aplicadas por especialistas que o trabalho das classificações pode ser refinado.

No entanto, poucos trabalhos comparam especificamente as regras de classificação por GEOBIA. Além da grande quantidade de atributos quantificáveis, há inúmeros impactos nos resultados quando se promove pequenas variações nesses parâmetros de classificação. Considerando que a comparação de regras de segmentação e classificação por GEOBIA contribui para ampliar a acurácia dos procedimentos, o objetivo do artigo é analisar duas classificações de 
vias pavimentadas de Marília - SP, por técnicas de GEOBIA resultantes de dois operadores distintos.

\section{MATERIAIS E MÉTODOS}

\subsection{Sensoriamento Remoto}

Para Singh (1989), a detecção de mudanças é o processo de identificação de diferenças no estado de um objeto ou fenômeno, em distintos períodos. É útil em diversas aplicações, como: análise das transformações na cobertura da terra, avaliação do desmatamento, mudanças sazonais na vegetação e monitoramento de desastres. O Sensoriamento Remoto é uma das técnicas usadas na geografia para localizar, classificar e estimar essas alterações (CURRAN, 1987).

De acordo com Cutter et al. (2002), o conhecimento geográfico requer a capacidade de compreender as mudanças de escala dos fenômenos e suas representações espaciais. Também pode ser associado à compreensão dos efeitos: da distância, direção, orientação, distribuição e padrão; da natureza das hierarquias espaciais; do significado da adjacência e dos vizinhos mais próximos, entre outros.

Segundo Herold et al. (2003), informações detalhadas sobre a cobertura urbana da terra são essenciais para o gerenciamento e planejamento urbano. A interpretação de fotografias aéreas é usada há décadas para essas finalidades e, seu processo de análise, frequentemente utiliza as relações hierárquicas dos elementos mostrados na Figura 1.

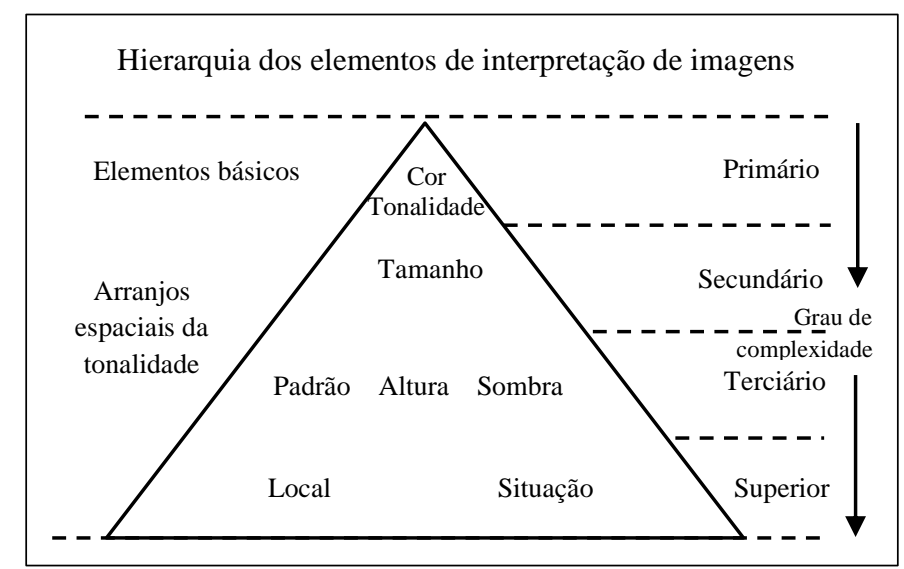

Figura 1 - Elementos de interpretação de imagens Fonte: Estes et al., 1983.

Embora um usuário experiente possa utilizar esses elementos na interpretação visual, esse conhecimento especializado não é facilmente traduzido para o processamento digital de imagens de satélites. Desenvolvimentos recentes na classificação de imagens "orientada ao objeto", baseada na segmentação de imagens, permite explorar as características espaciais detalhadas das imagens de alta resolução espacial e reduzir essas limitações (HEROLD et al., 2003). 
A análise urbana provavelmente representa o maior desafio do Sensoriamento Remoto em termos de classificação da cobertura da terra e seu desenvolvimento está intrinsecamente relacionado com o aumento da resolução das imagens de satélites. No entanto, devido à dificuldade de discriminação dos objetos com base nos dados espectrais no meio urbano, a qualidade das classificações, análises e modelagem depende da efetiva integração de informações auxiliares (LONGLEY, 2002).

Ainda de acordo com esse autor, o Sensoriamento Remoto não representa uma panaceia para a Geografia, mas melhora a qualidade dos dados empregados na análise espacial urbana. $\mathrm{O}$ desafio para os pesquisadores que usam fontes auxiliares e sintaxe espacial para melhorar a classificação das imagens consiste na avaliação crítica das metáforas que os geógrafos usam para entender a forma e a função urbana. O desenvolvimento tecnológico contribui para essas investigações, pois permite aumentar a complexidade da representação e da modelagem dos sistemas e, ao mesmo tempo, capacita os usuários a medir e monitorar essa complexidade.

\subsection{Análise de imagens baseadas em objetos geográficos (GEOBIA)}

O Sensoriamento Remoto desenvolve técnicas capazes de fornecer imagens da superfície do planeta por meio da detecção e medição quantitativa das respostas espectrais dos objetos da superfície (MENESES; ALMEIDA, 2012). O processo ocorre em duas fases: a primeira diz respeito à aquisição de dados através de sensores remotos alocados em aviões e satélites e, a segunda, está relacionada à interpretação humana dos dados e extração de informações dos objetos e feições (LUCHIARI, 2004).

O avanço da tecnologia dos Sensores Orbitais é uma resposta ao nível de detalhamento necessário aos estudos intraurbanos, juntamente com novos métodos semiautomáticos para a extração de informações, como, por exemplo, a cobertura da terra (ARAUJO; LUCHIARI, 2016).

A pesquisa em GEOBIA tem demonstrado sua eficácia, especialmente - mas não exclusivamente -, quanto à classificação de imagens de Sensoriamento Remoto de alta resolução (BLASCHKE; HAY, 2001). Destaca-se por apresentar uma unidade de processamento de imagem inovadora, transcendendo o nível do pixel e atuando com o objeto geográfico. Assim, além da assinatura espectral, também são considerados: textura, padrões geométricos, contexto, relações topológicas e de vizinhança. Esse conjunto de critérios permite a simulação de processos cognitivos humanos utilizados nas interpretações visuais (CRUZ et al., 2009, BLASCHKE; KUX, 2007, NAVULUR, 2006).

As duas principais fases de processamento são: a determinação dos objetos e a classificação. A determinação dos objetos exige um procedimento prévio de segmentação. Essa 
divisão automática da imagem em regiões homogêneas é realizada de acordo com parâmetros de escala, que delimita os objetos conforme as características dos pixels vizinhos (DESCLÉE et al., 2006).

Na classificação, são atribuídas informações semânticas aos objetos geográficos. Visa descrevê-los de forma lógica, considerando os atributos que diferenciam os objetos entre si (BLASCHKE; KUX, 2007). Os dois principais procedimentos de classificação são: 1) uso de funções de associação, como o classificador de vizinho mais próximo (Nearest Neighbourhood); 2) uso de regras, estabelecidas entre os atributos e os parâmetros, que dependem do conhecimento prévio do operador sobre o objeto-alvo. Observa-se que as funções de associação são raramente transferíveis por dependerem da fase de treinamento e de amostragem da imagem (HODGSON et al., 2003).

O fluxograma de procedimentos da pesquisa está apresentado na Figura 2. Os materiais utilizados nas classificações foram:

- Imagem WordView II (RGB e NIR) de Marília, de 18/08/2010: resolução espacial de 2m nas bandas multiespectrais e de $0,6 \mathrm{~m}$ na banda pancromática, todas com resolução radiométrica de 11 bits por pixel;

- Programa eCognition Developer 8.7 para classificação das vias;

- Programa ArcGis 9.3 para a avaliação quantitativa, baseada no cálculo do índice kappa, e qualitativa, através de análise visual.

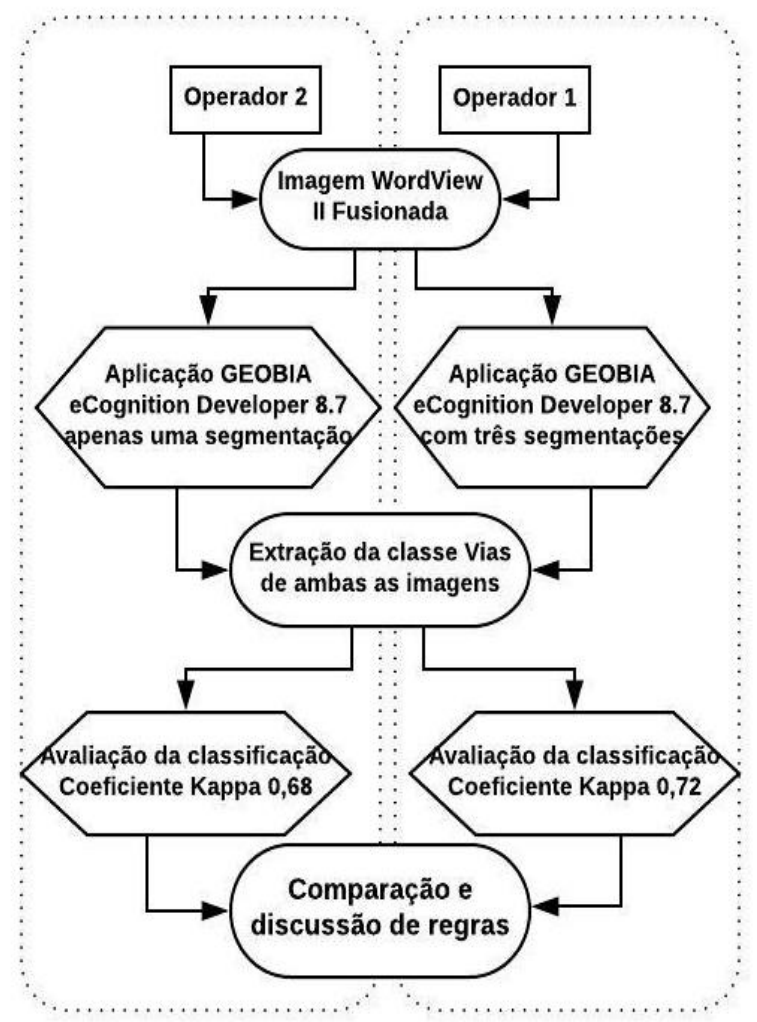

Figura 2 - Fluxograma metodológico. 
Após a definição das classes da imagem, foram selecionados os parâmetros de segmentação do programa eCognition: escala, compacidade (compactness) e forma (shape). O parâmetro escala representa a tolerância à heterogeneidade dentro de um segmento e define seu tamanho relativo. Quanto maior for o valor da escala, maiores deverão ser os segmentos dos objetos (SILVA et al., 2016, BAATZ; SCHÄPE, 2000).

A forma é composta pelos parâmetros de compacidade dos objetos e suavidade das bordas. São valores complementares que representam a complexidade espacial. A compacidade ressalta o aspecto geométrico do objeto e, a suavidade, a uniformidade dos seus contornos. De maneira geral, quanto maior o valor atribuído à compacidade, melhor será a segmentação de objetos que caracterizam as construções antrópicas. Os valores elevados de suavidade tendem a aumentar a eficiência da segmentação de objetos que representam as feições naturais, como vegetação e hidrografia (KRESSLER; STEINNOCHER, 2006).

Os atributos quantificáveis são as variáveis da imagem de satélite solicitadas pelo programa eCognition. Permitem a identificação das características e dos comportamentos de um objeto da imagem, os quais possibilitam a individualização das diferentes classes. Os atributos utilizados estão descritos na Tabela 1.

Tabela 1 - Atributos quantificáveis e variáveis do eCognition.

\begin{tabular}{|c|c|c|c|}
\hline Tipo de atributo & Atributo quantificável & Formulação matemática & Descrição \\
\hline \multirow{4}{*}{ Espectral } & $\begin{array}{c}\text { Índice de Vegetação por } \\
\text { Diferença Normalizada (NDVI) }\end{array}$ & $\mathrm{NDVI}=$ média $\left(\frac{N I R-R E D}{N I R+R E D}\right)$ & $\begin{array}{c}\text { O NDVI é um valor entre } \\
-1.0 \mathrm{e}+1.0\end{array}$ \\
\hline & Brightness & $\mathrm{B}=\frac{1}{n_{v i s}} \sum_{i=1}^{n_{v i s}} C_{i(v i s)}^{\smile}$ & $\begin{array}{c}\text { B é o brilho médio de um } \\
\text { objeto e } C_{i(v i s)} \text { é a soma } \\
\text { de todos os valores médios } \\
\text { de brilho nas bandas } \\
\text { visíveis dividido pelo } \\
\text { número correspondente de } \\
\text { bandas visíveis } n_{v i s}\end{array}$ \\
\hline & Rectangular Fit & $\begin{array}{l}\mu_{R}(x)=\left\{1-\frac{1-x}{1-T}\right. \\
\text { for } 0 \leq \mathrm{X}-\mathrm{T} \geq 1-\mathrm{T}\end{array}$ & $\begin{array}{l}\text { Descreve quão bem um } \\
\text { objeto de imagem (x) se } \\
\text { encaixa em um retângulo } \\
\text { (T). Valores entre } 1 \\
\text { (encaixe perfeito) e } 0 \text { (não } \\
\text { encaixa). }\end{array}$ \\
\hline & Shape Length/Width & $\frac{\text { Length }}{\text { Width }}$ & $\begin{array}{c}\text { Comprimento do objeto de } \\
\text { imagem } \\
\text { Largura do objeto de } \\
\text { imagem. }\end{array}$ \\
\hline
\end{tabular}

Fonte: Adaptado de Araújo e Queiroz, 2018. 


\subsection{Regras de classificação}

As regras de classificação das vias foram representadas por árvores de processos, como mostram as Figuras 3 e 4, e ilustram os procedimentos dos dois intérpretes para extrair as feições geográficas da cidade de Marília. As classes utilizadas por ambos foram: vegetação intra e extra urbana, corpos hídricos, sombras e tipos de telhados, diferenciados pela composição dos respectivos materiais (cerâmica, cimento e alumínio).

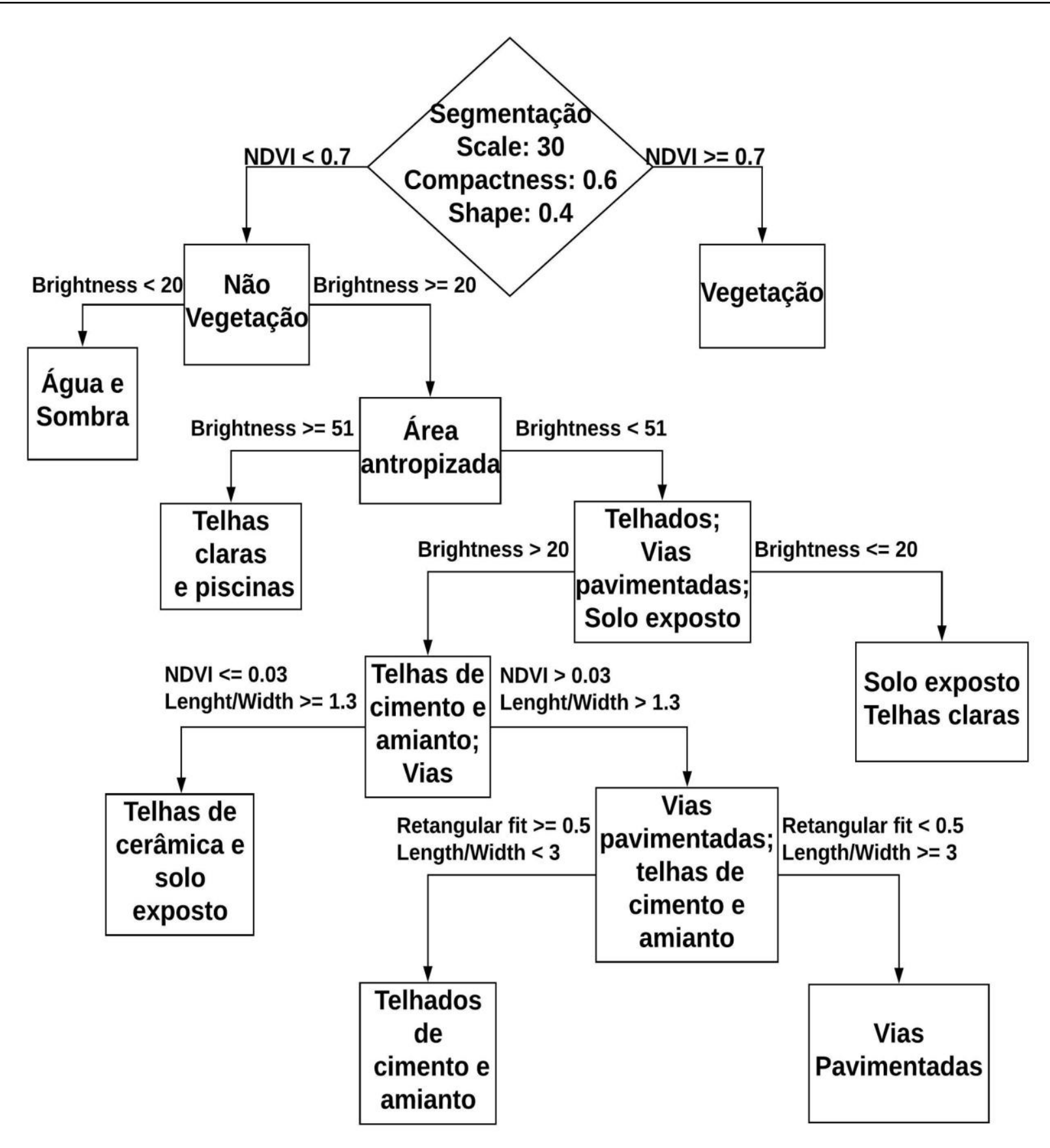

Figura 3 - Árvore de decisões do operador 1. 


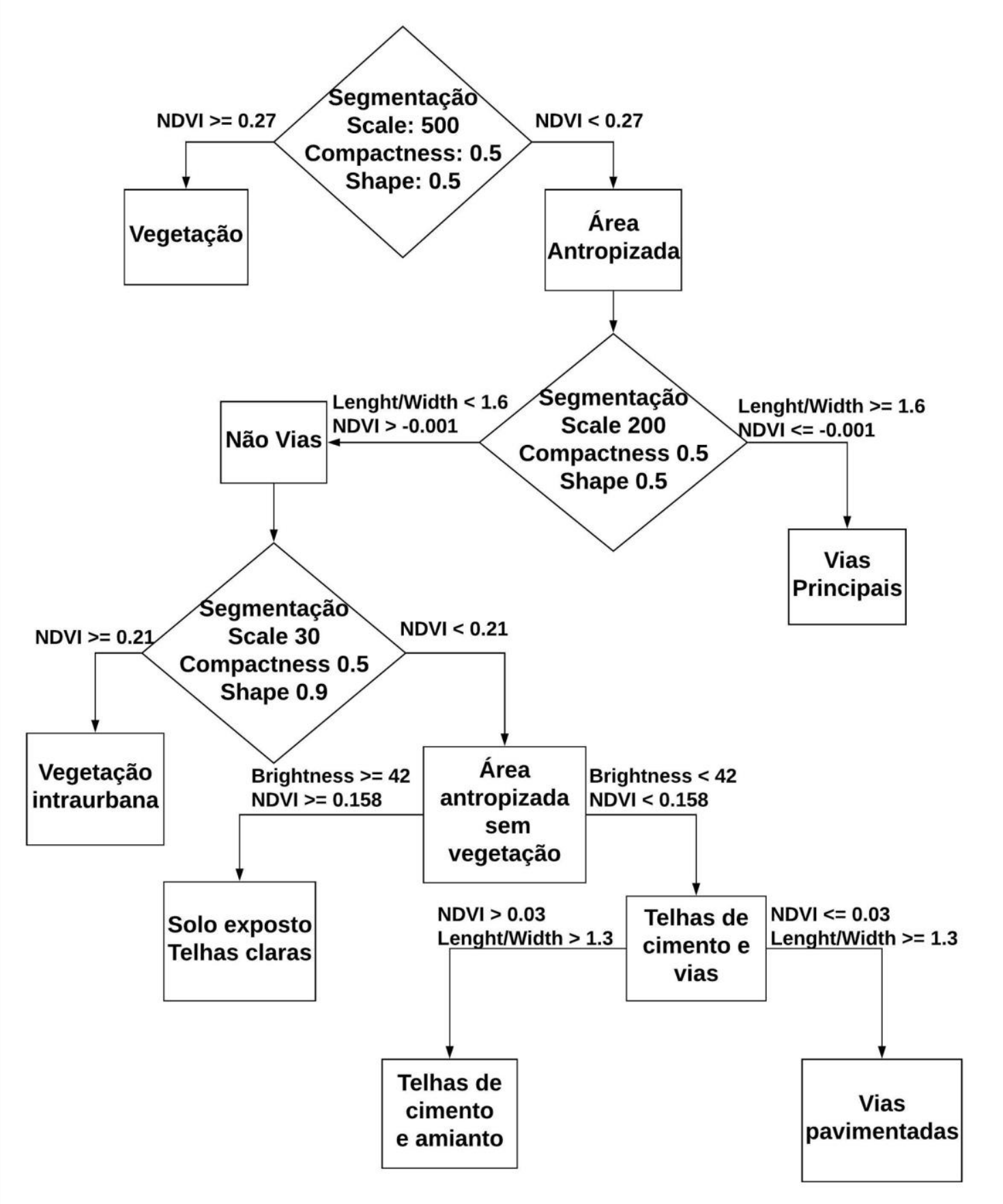

Figura 4 - Árvore de decisões do operador 2.

\subsection{COEFICIENTE KAPPA}

O coeficiente kappa é uma medida estatística para avaliar a exatidão das classificações. Mede o nível de concordância entre dois conjuntos de dados e determina a acurácia das análises (FITZGERALD; LEES, 1994). Seu uso é muito difundido, pois uma significativa quantidade de pesquisas adota o coeficiente kappa para avaliar a qualidade das classificações de imagens de Sensoriamento Remoto (ARAUJO; QUEIROZ, 2018, BELGIU et al., 2014, FRANCISCO; ALMEIDA, 2013, PAL, 2007, PINHO, 2006, FITZGERALD; LEES, 1994). O valor estimado da estatística kappa é dado pela equação (1): 


$$
k=P o-P i / 1-P i
$$

Onde:

Po é a proporção de concordância total das classificações,

$P i$ é a soma das probabilidades independentes.

\section{RESULTADOS}

Como esperado, as classificações originárias dos diferentes processos apresentaram resultados visual e estatisticamente distintos (Figura 5 A e B).

A primeira árvore de processos (Figura 3) possibilitou a extração das vias a partir de objetos pequenos, devido aos parâmetros de segmentação da imagem adotados início do processo (parâmetro de escala $=30)$. O resultado foi a redução da linearidade das vias (figura 5A), a diminuição da área de abrangência e da força de concordância da classificação (kappa de 0,68).

A segunda árvore de processos (Figura 4) apresentou maior nível de generalização das vias pavimentadas e, consequentemente, maior área classificada. Os parâmetros de segmentação da segunda árvore de decisão aumentaram a continuidade espacial dos objetos de interesse e permitiram a extração das maiores vias pavimentadas como objetos únicos (rodovias e avenidas), como demonstra a figura 5B (kappa de 0,72$)$.

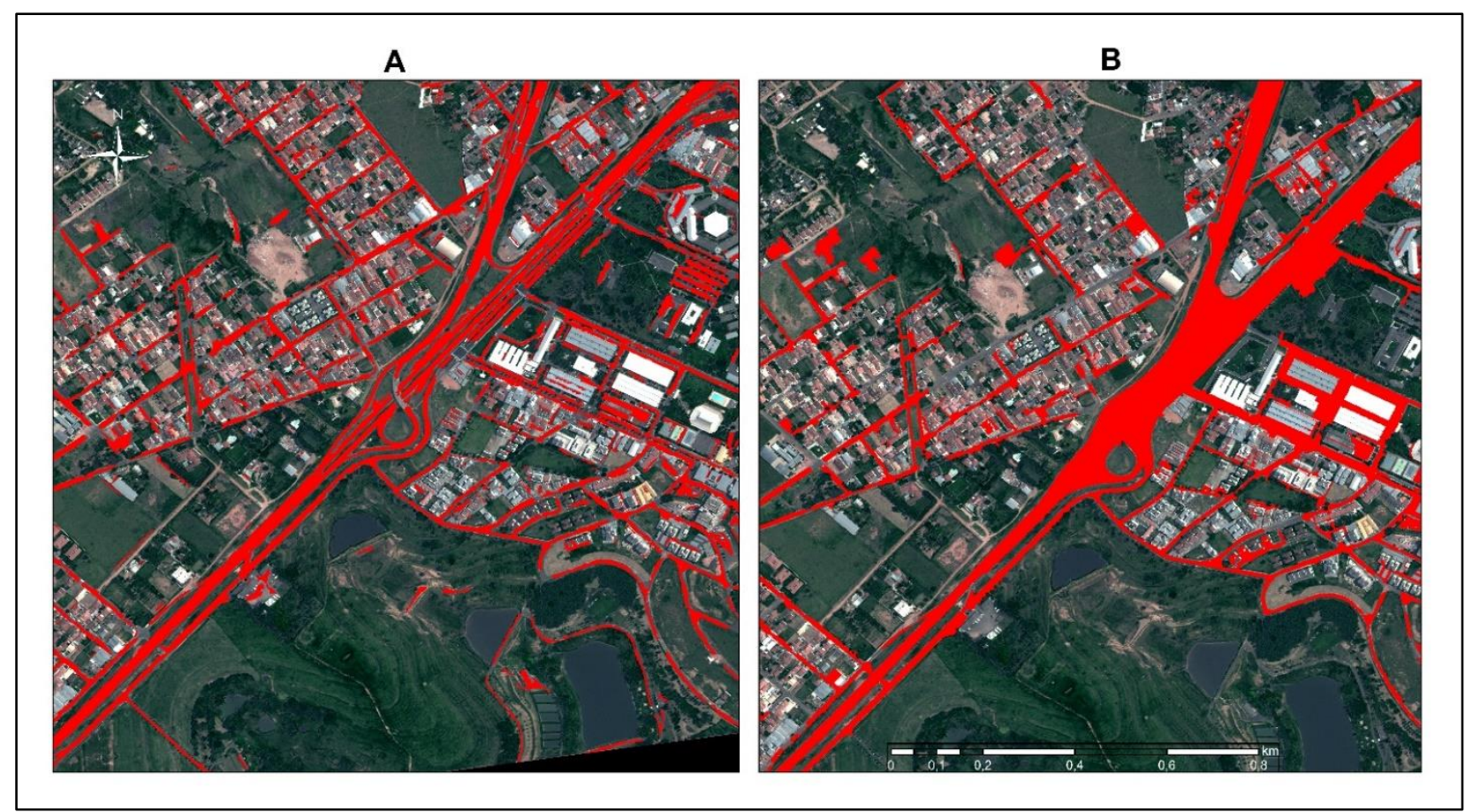

Figura 5 - Amostras da classificação final dos operadores 1 (5A) e 2 (5B). 
A primeira segmentação representou a diferença mais significativa entre os dois operadores. Enquanto o primeiro classificou todos os objetos a partir de uma única segmentação (mais específica), o segundo optou por três segmentações (mais genéricas).

O primeiro operador optou por segmentar a imagem com parâmetros mais restritivos, que resultou em objetos menores (parâmetros de scale $=30$, compactness $=0,6$ e shape $=0,5$ ). $\mathrm{O}$ segundo operador segmentou a imagem em três fases: na primeira, extraiu os objetos maiores (scale $=500$, compactness $=0,5$ e shape $=0,5)$; na segunda, utilizou a área antropizada como filtro e segmentou os objetos de tamanho médio (scale $=200$, compactness $=0,5$ e shape $=0,5)$ e, na terceira, segmentou os objetos de menor tamanho ainda não classificados (parâmetros de scale = 30 , compactness $=0,5$ e shape $=0,9$ ).

A diferenciação inicial da segmentação entre os operadores impactou todos os processos subsequentes, uma vez que a escala utilizada determinou o tamanho dos objetos criados e, consequentemente, a informação semântica contida em cada um. De forma geral, os atributos quantificáveis utilizados foram os mesmos (NDVI, lenght/width e o brightness), com exceção do retangular fit, utilizado apenas pelo primeiro operador.

A amplitude dos valores, no entanto, bem como a combinação entre 2 atributos quantificáveis variou. Para classificar as áreas de vegetação e áreas antropizadas, por exemplo, ambos os operadores utilizaram o NDVI (Normalized Difference Vegetation Index), no entanto, a amplitude dos valores variou entre 0,7 (operador 1) e 0,27 (operador 2). A combinação entre 2 atributos na última fase de classificação foi distinta, enquanto o primeiro operador associou retangular fit com lenght/width, o segundo operador utilizou NDVI e lenght/width.

Desta maneira, as duas árvores de processos expressaram variações na seleção dos atributos e nos valores dos parâmetros nas fases de classificação e segmentação, resultando em distintas classificações de vias pavimentadas, dos pontos de vista qualitativo e quantitativo. As Tabelas 2 e 3 apresentam as matrizes de confusão que foram produzidas para avaliar as classificações de ambos os operadores (500 pontos aleatórios). 
Tabela 2 - Matriz de confusão e índice kappa (Operador 1).

\begin{tabular}{cccc}
\hline$\#$ & Vias pavimentadas & Não vias & Total \\
\hline Vias pavimentadas & 205 & 62 & 267 \\
Não vias & 9 & 224 & 233 \\
Total & 214 & 286 & 500
\end{tabular}

Concordância observada: $84,2 \%$

Probabilidade de concordância ao acaso: 49,8\%

Coeficiente kappa: $68,13 \%$

Tabela 3 - Matriz de confusão e índice kappa (Operador 2).

\begin{tabular}{cccc}
\hline$\#$ & Vias pavimentadas & Não vias & Total \\
\hline Vias pavimentadas & 211 & 56 & 267 \\
Não vias & 24 & 209 & 233 \\
Total & 235 & 265 & 500
\end{tabular}

Concordância observada: $85,8 \%$

Probabilidade de concordância ao acaso: 49,6\%

Coeficiente kappa $\mathbf{7 1 , 8 \%}$

\section{DISCUSSÃO}

O contexto desta pesquisa está associado à conjuntura de desenvolvimento tecnológico recente: 1) disponibilidade de imagens de satélite de alta resolução, 2) evolução de técnicas semiautomatizadas de classificação, 3) redução da intervenção humana no processamento e 4) reprodução e compartilhamento dos procedimentos de análise. Foi inspirada pelo trabalho de Belgiu et al. (2014), que avaliaram a variabilidade das classificações realizadas por especialistas independentes. Os resultados dos pesquisadores austríacos mostraram diferenças estatisticamente significativas entre os operadores, mas as classificações obtiveram resultados satisfatórios quando transferidas para outra área, sem modificação das regras desenvolvidas.

Os resultados obtidos pela classificação das vias de Marília por dois operadores independentes corroboraram as afirmações de Herold et al. (2003) de que os princípios de interpretação visual não se transferem com facilidade para o processamento digital de imagens de satélites e, sobretudo, de que a segmentação de imagens contribui para explorar as características espaciais detalhadas das imagens urbanas de alta resolução espacial. Também foram considerados coerentes com as observações de Cutter et al. (2002), segundo os quais o conhecimento geográfico 
estaria também relacionado à compreensão dos efeitos da distância, direção, orientação, distribuição e padrão dos fenômenos. E ratificam as considerações de Longley (2002), de que a análise urbana representa o maior desafio do Sensoriamento Remoto no que se refere à classificação da cobertura da terra e que a melhoria da sua qualidade depende da integração de informações auxiliares.

Os resultados obtidos na classificação das vias da Marília foram qualitativa e quantitativamente distintos. Quando comparados, evidenciaram a influência da subjetividade do analista no processo e na acurácia da classificação, em consonância com as observações de Gardin et al. (2010). As diferenças decorreram, principalmente, pela escolha das regras de classificação, das configurações dos limites e dos parâmetros de segmentação nos vários níveis hierárquicos.

Do ponto de vista qualitativo, ambas as classificações pelo método GEOBIA obtiveram baixa confusão entre classes semelhantes. Exemplo: os telhados de cimento amianto e as vias pavimentadas somente foram separados com os critérios de geometria, pois ambas as classes possuem assinaturas espectrais semelhantes. Esse aspecto corrobora as observações de inúmeros pesquisadores (MYINT et al., 2011, PLATT; RAPOZA, 2008, WHITESIDE; AHMAD, 2005, ORUC et al., 2004, CIVCO et al., 2002, WILLHAUCK, 2000).

Em relação à análise quantitativa das classificações, uma amostra de 500 pontos aleatórios de verdade de campo foi usada para calcular o índice kappa. Foi estratificada, de forma que aproximadamente $50 \%$ do total de pontos foram classificados como vias e o restante como não vias. Para a interpretação dos resultados adotou-se a qualificação dos valores do índice kappa proposta por Landis e Koch (1977), reproduzida na Tabela 4 (variam entre 0 e 1).

Tabela 4 - Interpretação dos valores do índice kappa.

\begin{tabular}{cc}
\hline Valores de kappa & Interpretação \\
\hline$<0$ & Sem concordância \\
$0-0,19$ & Pouca concordância \\
$0,2-0,39$ & Concordância razoável \\
$0,4-0,59$ & Concordância média \\
$0,6-0,79$ & Concordância considerável \\
$0,8-1,0$ & Concordância excelente \\
\hline
\end{tabular}

Fonte: Landis e Koch (1977), p. 165.

Assim, considerando que a classificação do operador 1 obteve 0,68 e que o operador 2 alcançou 0,72, a interpretação foi de que houve concordância considerável entre os intérpretes, de acordo com a tabela 4 .

Por outro lado, é possível refletir sobre a limitação do coeficiente kappa para avaliar a acurácia das classificações. O paradoxo, demonstrado nos resultados, é que apesar dos valores de concordância terem sido mais altos (84,2\% e 85,8\%), os do índice kappa foram mais baixos (68,1\% 
e 71,8\%). Uma possível explicação para o fato é que o kappa considera a probabilidade de classificações aleatórias e, quando são analisadas apenas duas classes (vias pavimentadas e não vias), a probabilidade de se classificar corretamente um objeto é de aproximadamente 50\%. Ainda que essa penalização das probabilidades de concordâncias aleatórias do kappa tenha sido extensivamente discutida (HSU; FIELD, 2003), ainda não há índices gerais, com prerrogativas semelhantes, que facultem sua substituição.

Outro elemento importante estaria relacionado à interpretação dos valores de kappa. A proposta por Landis e Koch (1977) é frequentemente adotada para analisar a concordância entre os conjuntos de dados, embora tenha sido elaborada há mais de vinte anos. Nesse sentido, acredita-se que sua revisão seria relevante para atender aos novos métodos de classificação, como GEOBIA, pois costumam apresentar valores elevados (acima de 0,8 ).

\section{CONSIDERAÇÕES FINAIS}

A pesquisa comparou duas classificações de vias pavimentadas de Marília - SP, por técnicas de GEOBIA, resultantes de dois operadores independentes. Como esperado, constatou-se que as classificações apresentaram diferenças estatísticas, em decorrência dos distintos critérios empregados nas regras de segmentação e classificação para a extração final das vias pavimentadas.

O coeficiente kappa e a análise visual foram utilizados para comparar ambas as classificações. Os índices kappa de 0,68 e 0,72 apontaram para uma considerável concordância entre os operadores. Os resultados também destacaram a importância dos critérios de segmentação e dos parâmetros de escala de análise, principais diferenças entre os operadores, na classificação dos objetos geográficos.

Além disso, acredita-se que essas regras e parâmetros de classificação podem ser utilizados como referências para a classificação de vias pavimentadas em outras áreas de estudo, uma vez que o método se baseia no conhecimento a priori dos objetos-alvos (BELGIU et al., 2014). O compartilhamento das regras de classificação e parâmetros de segmentação poderá facilitar o trabalho de classificações posteriores, bem como possibilitar seu aperfeiçoamento (MAHDAVI et al., 2018).

\section{AGRADECIMENTOS}

Ao CNPq, pelo financiamento da pesquisa (Projeto Universal - Processo: 408077/2016-6). 


\section{REFERÊNCIAS}

ALMEIDA, C. M. Aplicação dos sistemas de sensoriamento remoto por imagens e o planejamento urbano regional. Revista eletrônica de Arquitetura e Urbanismo (USJT), São Paulo, n. 3, p. 98$123,2010$.

ARAUJO, A. S.; LUCHIARI, A. Elaboração de mapa de cobertura da terra intraurbana com base em análise baseada em objetos (OBIA) para inferências sobre o uso na cidade de Marília/SP. Geografia, Rio Claro, v. 41, n. 2, p. 241-260, 2016.

ARAUJO, A. S.; QUEIROZ, A. P. Spatial Characterization and Mapping of Gated Communities. International Journal of Geo-Information, Vienna, v. 7, n. 7, p. 1-22, 2018.

BAATZ, M.; SCHÄPE, A. Multi resolution Segmentation: an optimum approach for high quality multi scale image segmentation. Angewandte Geographische Informationsverarbeitung, Karlsruhe, n. 12, p. 12-23, 2000.

BLASCHKE, T.; HAY, G. Object-oriented image analysis and scale-space: theory and methods for modeling and evaluating multi-scale landscape structure. The International Archives of the Photogrammetry, Remote Sensing and Spatial Information Science, n. 34, 22-29, 2001.

BLASCHKE, T.; KUX, H. Sensoriamento Remoto e SIG Avançados: novos sistemas sensores métodos inovadores. 2. ed. Oficina de Textos, 2007. 304p.

BELGIU, M.; DRĂGUT, L.; STROBL, J. Quantitative evaluation of variations in rule-based classifications of land cover in urban neighbourhoods using WorldView-2 imagery. ISPRS Journal of Photogrammetry and Remote Sensing, v. 87, p. 205-215, 2014.

CIVCO, D. L.; HURD, J. D.; WILSON, E. H.; SONG, M.; ZHANG, Z. A comparison of land use and land cover change detection methods. Paper presented at the annual conference of the ASPRSACSM Annual Conference, Washington, v. 22, p. 7-11, 2002.

CRUZ, C. B. M.; ROSÁRIO, L. D.; ABREU, M. D.; ALMEIDA, P. D.; VICENS, R. S.; CRONEMBERGUER, F. Classificação Orientada a Objetos na Geração do Mapa de Uso e Cobertura da Terra do estado do Rio de Janeiro. In: SIMPÓSIO BRASILEIRO DE SENSORIAMENTO REMOTO, 14., Natal. Anais... Natal, 2009. p. 25-30.

CURRAN, P. J. Remote sensing methodologies and geography, International Journal of Remote Sensing, v. 8, n. 9, p. 1255-1275, 1987.

CUTTER, S. L.; GOLLEDGE, R.; GRAF, W. L. The big questions in geography, The Professional Geographer, v. 54, n. 3, p. 305-317, 2002.

DESCLÉE, B.; BOGAERT, P.; DEFOURNY, P. Forest change detection by statistical object-based method. Remote Sensing of Environment, v. 102, n. 1-2, p. 1-11, 2006.

ESTES, J. E.; HAJIC, E. J.; TINNEY, L. R. Fundamentals of image analysis: Analysis of visible and thermal infrared data. In: COLWELL, R.N. Manual of Remote Sensing, American Society of Photogrammetry, 1983. p. 987-1124.

FITZGERALD, R. W.; LEES, B. G. Assessing the classification accuracy of multisource remote sensing data. Remote Sensing of Environment, v. 47, n. 3, p. 362-368, 1994. 
FRANCISCO, C. N.; ALMEIDA, C. M. Avaliação de desempenho de atributos estatísticos e texturais em uma classificação de cobertura da terra baseada em objeto. Boletim de Ciências Geodésicas, Curitiba, v. 18, n. 2, p.302-326, 2013.

GARDIN, S.; VAN LAERE, S. M.; VAN COLlIE, F. M. B.; ANSEEL, F.; DUYCK, W.; DE WULF, R. R.; VERBEKE, L. P. C. Remote sensing meets psychology: a concept for operator performance assessment. Remote Sensing Letters, v. 2, n. 3, p.251-257, 2011.

HAY, G. J.; CASTILLA, G. Geographic Object-Based Image Analysis (GEOBIA): A new name for a new discipline. In: ADDINK, E. Object-based image analysis. Berlin: Springer-Heidelberg, 2008, cap. 4. p. 75-89.

HEROLD, M.; LIU, X.; CLARKE, K. C. Spatial metrics and image texture for mapping urban land use. Photogrammetric Engineering \& Remote Sensing, v. 69, n. 9, p. 991-1001, 2003.

HODGSON, M.; JENSEN, J. R.; TULLIS, J. A.; RIORDAN, K.; ARCHER, C. M. Synergistic use of lidar and color aerial photography for mapping urban parcel imperviousness. Photogrametric Engineering and Remote Sensing, v. 69, n. 9, p. 973- 980, 2003.

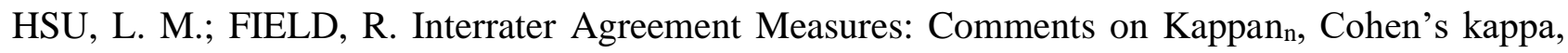
Scott's $\pi$, and Aickin's $\alpha$. Understanding Statistics, New Jersey, v. 2, n. 3, p. 205-2019, 2003.

KRESSLER, F. P.; STEINNOCHER, K. Image data and LIDAR-an ideal combination matched by object oriented analysis. Geographic Object-Based Image Analysis, Salzburg, v. 36, n. 1, p. 3660, 2006.

LANDIS, R. J.; KOCH, G. G. The Masurement of Observed Agreement for Categorial Data. Biometrics, v. 33, n. 1, p.159-174, 1977.

LONGLEY, P. A. Geographical Information Systems: will developments in urban remote sensing and GIS lead to 'better' urban geography? Progress in Human Geography, v. 26, n. 2, p. 231239, 2002.

LUCHIARI, A. Imagens da metrópole. In: CARLOS, A. A.; OLIVEIRA, A. U. (Org.) Geografias de São Paulo: representação e crise da metrópole. São Paulo: CONTEXTO, 2004, cap. 3, p. 231246.

MAHDAVI, S.; SALEHI. B.; GRANGER, J.; AMANI, M.; BRISCO, B.; HUANG, W. Remote sensing for wetland classification: A comprehensive review. GIScience \& Remote Sensing, v. 55, n. 5, p. 623-658, 2018.

MENESES, P. R.; ALMEIDA, T. Introdução ao processamento de imagens de Sensoriamento Remoto. 1. ed. Brasília: Conselho Nacional de Desenvolvimento Científico e Tecnológico, 2012. 266p.

MYINT, S. W.; GOBER, P.; BRAZEL, A.; GROSSMAN-CLARKE, S.; WENG, Q. Per-pixel vs. object-based classification of urban land cover extraction using high spatial resolution imagery. Remote Sensing of Environment, v. 115, n. 5, p.1145-1161, 2011.

NAVULUR, K. Multispectral image analysis using the object-oriented paradigm. 1. ed, Boca Raton: Taylor \& Francis Group, 2006. 240p. 
ORUC, M.; MARANGOZ, A. M.; BUYUKSALIH, G. Comparison of pixel-based and objectoriented classification approaches using Landsat-7 ETM spectral bands. In: ISPRS CONGRESS, 20., 2004. ProceedingS... Istanbul, 2004. p. 19-23.

PAL, M. Random forest classifier for Remote Sensing classification. International Journal of Remote Sensing, v. 26, n. 1, p. 217-222, 2007.

PINHO, C. M. D. Análise orientada a objetos de imagens de satélites de alta resolução espacial aplicada à classificação de cobertura do solo no espaço intra-urbano: o caso de São José dos Campos. 2006. 180 f. Dissertação (Mestrado em Sensoriamento Remoto) - Instituto Nacional de Pesquisas Espaciais, São José dos Campos, 2006.

PLATT, R. V.; RAPOZA, L. An evaluation of an object-oriented paradigm for land use/land cover classification. The Professional Geographer, v. 60, n. 1, p. 87-100, 2008.

SIlva, A. A.; OliveirA, A. P. G.; PARANHOS FILHO, A. C.; GRIGIO, A. M. Uso do eCognition 8.9 para classificação de Imagem de satélite de alta resolução. 1. ed., Mossoró: EDUERN, 2016, 106p.

SINGH, A. Digital change detection techniques using remotely-sensed data. International Journal of Remote Sensing, v. 10, n. 6, p. 989-1003, 1989.

WILLHAUCK, G. Comparison of object-oriented classification techniques and standard image analysis for the use of change detection between SPOT multispectral satellite images and aerial photos. In: ISPRS CONGRESS, 19., 2000, Amsterdam. Proceedings... Amsterdam, 2000. p. 4150 .

WHITESIDE, T.; AHMAD, W. A comparison of object-oriented and pixel-based classification methods for mapping land cover in northern Australia. Spatial intelligence, innovation and praxis: The national biennial conference of the Spatial Sciences Institute, Australia, 2005, p. 1225-1231. 